\title{
A Method for Calculating and Continuing Static Solutions for Flexible Multibody Systems
}

\author{
J. P. Meijaard \\ Precision Engineering \\ University of Twente \\ PO Box 17, 7500 AE Enschede, The Netherlands \\ J.P.Meijaard@utwente.nl
}

\begin{abstract}
This presentation describes some modifications in the calculation of static solutions in the program SPACAR and the implementation of an arc continuation method to calculate a branch of static solutions for varying parameters. The equations to be solved are stated in a descriptor form, which can be viewed as the equations of motion written as differential-algebraic equations with the time-dependent terms removed. The arc continuation method is of the familiar predictor-corrector type. The equations of motion are linearized around found equilibria and reduced to a state-space description in the degrees of freedom and their rates.

The methods are applied to several examples: planar and spatial large deflection problems for beams, the buckling of a parallel leaf-spring guidance by misalignments and the large deflections and stability of a fluid-conveying tube. It is shown that the proposed methods can lead to higher robustness and efficiency.
\end{abstract}

Keywords: static solutions, differential-algebraic equations, linearized state-space equations, arc continuation.

\section{Introduction}

In the analysis of multibody dynamic problems, it is often useful to know the equilibrium configurations and the way in which these configurations change if a loading parameter changes. These solutions can often be considered initial quasistatic approximations of the true motion of a system if the loads change relatively slowly with respect to the eigenfrequencies of the system. Corrections can then be made with the aid of linearized dynamic equations. So here, a method for finding and continuing static equilibrium solutions of a flexible multibody system and obtaining the linearized equations in these equilibria is presented.

For the modelling of the multibody systems, use is made of a formalism based on finite element techniques that has been developed over several years $[1,2,3]$. This formalism introduces, besides nodal coordinates, so-called generalized strains for each element and their energetically dual quantities, generalized stresses.

Static solutions can be found by putting all velocities and accelerations in the equations of motion equal to zero and solving the resulting set of ordinary, generally non-linear, equations. In order to find nearby static solutions after some initial solution has been found and a system parameter is changed, so a branch of static solutions can be traced, continuation methods have been developed. These methods consist of an explicit predictor, which estimates a new solution some distance away from a current solution, and an iterative corrector, which determines a more accurate solution on the branch of equilibria. One of the first to apply these methods was Haselgrove [4]. Many fundamental ideas for continuation are contained in this article. An early application to structural mechanics was made by Riks $[5,6]$ and Wempner [7], where an auxiliary equation that describes a hyperplane perpendicular to the tangent vector of a known solution was used to define a step size. Another auxiliary condition was used by Crisfield [8], whereas Fried [9] proposed a search direction orthogonal to the present level curve.

An application of continuation methods to the problem of finding equilibrium solutions for flexible multibody systems was reported by Cardona and Huespe [10]. Their method resembles somewhat the proposed model here, in that Lagrangian multipliers, analogous to the generalized stresses used here, are included in the set of equations that has to be solved, but their formulation does not include generalized strains. Although the system of equations to be solved in the present method is larger, the system matrix remains sparse and the method has generally better convergence properties. Here, in addition to finding the equilibrium solutions, the linearized equations of motion in equilibria are formulated in a set of minimal coordinates, that is, the degrees of freedom.

The structure of the paper is as follows. Section 2 describes a way to obtain stationary solutions for a multibody system including constraints. Section 3 describes a procedure to obtain the linearized equations of motion in an equilibrium configuration of the constrained system, expressed in independent coordinates. Section 4 describes a continuation 
method for calculating points along an arc of solutions if some combination of parameters is varied. In Section 5, applications to large-deflection problems of a planar and a curved spatial beam, the bucking of a parallel leaf-spring mechanism due to a misalignment and the deflection of a fluid-conveying tube due to the fluid flow are discussed. The paper ends with some conclusions.

\section{Finding Static Solutions}

As already said, the flexible multibody systems are modelled by finite elements, which have a number of nodes with nodal coordinates by which it can interact with the environment and be connected to other elements. For each element $e$ with nodal coordinates $\boldsymbol{x}^{e}$, a number of generalized strains $\boldsymbol{\varepsilon}^{e}$ are defined,

$$
\boldsymbol{\varepsilon}^{e}=\mathscr{D}^{e}\left(\boldsymbol{x}^{e}\right)
$$

where $\mathscr{D}^{e}$ is called the deformation function of the element. These generalized strains are invariant under rigid-body displacements of the element and describe the state of deformation of the element, so constraining them to be zero, or at least constant, enforces rigidity on the element. Generalized stresses $\boldsymbol{\sigma}^{e}$ are defined as energetically dual to the generalized strains, so the inner product of them with a virtual variation of the generalized strains represents the negative virtual work of the internal forces in the element, $\delta W_{\mathrm{i}}^{e}=-\boldsymbol{\sigma}^{e \mathrm{~T}} \delta \boldsymbol{\varepsilon}^{e}=-\boldsymbol{\sigma}^{e \mathrm{~T}} \mathscr{D}_{, \mathbf{x}}^{e} \delta \boldsymbol{x}^{e}$, where a subscript comma followed by a variable denotes a partial derivative with respect to that variable and a prefixed $\delta$ denotes a virtual variation. If these strains are not prescribed, either as constant values or as prescribed functions of time, the associated generalized stresses are given by constitutive relations, which are assumed to be linear, so they can be specified by a stiffness matrix $\boldsymbol{S}^{e}$, a damping matrix $\boldsymbol{S}_{\mathrm{d}}^{e}$ and an initial stress (pre-stress) $\boldsymbol{\sigma}_{0}^{e}$ as

$$
\boldsymbol{\sigma}^{e}=\boldsymbol{S}^{e} \boldsymbol{\varepsilon}^{e}+\boldsymbol{S}_{\mathrm{d}}^{e} \dot{\boldsymbol{\varepsilon}}^{e}+\boldsymbol{\sigma}_{0}^{e}
$$

A dot over a variable denotes a derivative with respect to time. The dynamic properties are described by a mass matrix and velocity-dependent convective terms, so the inertia force of the element is given by

$$
-\boldsymbol{M}^{e} \ddot{\boldsymbol{x}}^{e}-\boldsymbol{h}^{e}\left(\dot{\boldsymbol{x}}^{e}, \boldsymbol{x}^{e}\right)
$$

A system can be modelled by a number of elements of several kinds, which are linked by sharing nodal coordinates. Some coordinates and generalized strains can have prescribed values and applied forces can act on the system. The equations for the complete system can be obtained by the familiar finite-element assembly process. For static solutions, the inertia forces and also the viscous damping forces are absent.

The nodal coordinates can be divided into prescribed coordinates, which can either have a constant value or be prescribed functions of time and are denoted by $\boldsymbol{x}^{\mathrm{p}}$, and free coordinates, denoted by $\boldsymbol{x}^{\mathrm{f}}$. The dual forces are the reaction forces $f^{\mathrm{p}}$ and the applied forces $f^{\mathrm{f}}$. In the same manner, the generalized strains can be divided into prescribed strains $\boldsymbol{\varepsilon}^{\mathrm{p}}$, which can again have fixed values (mostly zero) or be prescribed functions of time, and free strains, $\boldsymbol{\varepsilon}^{\mathrm{f}}$. The dual generalized stresses are the reaction stresses $\sigma^{\mathrm{p}}$, also called Lagrangian multipliers for the imposed constraints, and the generalized stresses that follow from the constitutive equations, $\boldsymbol{\sigma}^{\mathrm{f}}$.

The equations that have to be satisfied are the equilibrium equations (equations of motion for dynamic problems), the constitutive relations for the generalized stresses dual to the free generalized strains and the compatibility relations. In a partitioned form, these can be written as

$$
\begin{gathered}
\left(\mathscr{D}_{, \boldsymbol{x}}^{\mathrm{pp}}\right)^{\mathrm{T}} \boldsymbol{\sigma}^{\mathrm{p}}+\left(\mathscr{D}_{, \boldsymbol{x}}^{\mathrm{fp}}\right)^{\mathrm{T}} \boldsymbol{\sigma}^{\mathrm{f}}-\boldsymbol{f}^{\mathrm{p}}=\mathbf{0}, \\
\left(\mathscr{D}_{, \boldsymbol{x}}^{\mathrm{pf}}\right)^{\mathrm{T}} \boldsymbol{\sigma}^{\mathrm{p}}+\left(\mathscr{D}_{, \boldsymbol{x}}^{\mathrm{ff}}\right)^{\mathrm{T}} \boldsymbol{\sigma}^{\mathrm{f}}-\boldsymbol{f}^{\mathrm{f}}=\mathbf{0}, \\
\boldsymbol{S}^{\mathrm{fp}} \boldsymbol{\varepsilon}^{\mathrm{p}}+\boldsymbol{S}^{\mathrm{ff}} \boldsymbol{\varepsilon}^{\mathrm{f}}+\boldsymbol{\sigma}_{0}^{\mathrm{f}}-\boldsymbol{\sigma}^{\mathrm{f}}=\mathbf{0}, \\
\mathscr{D}^{\mathrm{p}}\left(\boldsymbol{x}^{\mathrm{f}}, \boldsymbol{x}^{\mathrm{p}}\right)-\boldsymbol{\varepsilon}^{\mathrm{p}}=\mathbf{0} . \\
\mathscr{D}^{\mathrm{f}}\left(\boldsymbol{x}^{\mathrm{f}}, \boldsymbol{x}^{\mathrm{p}}\right)-\boldsymbol{\varepsilon}^{\mathrm{f}}=\mathbf{0} .
\end{gathered}
$$

The compatibility conditions define the system generalized strains $\boldsymbol{\varepsilon}$ as functions of the system nodal coordinates $\boldsymbol{x}$, where the system deformation function $\mathscr{D}$ is obtained as a direct sum of the element deformation functions. In the same way, the system constitutive relations are obtained from those of the elements in the form of a system stiffness matrix $S$. More complicated constitutive relations could be introduced if needed. Of course, the stresses dual to fixed and prescribed strains, $\boldsymbol{\sigma}^{\mathrm{p}}$, are not determined by constitutive relations, but follow from the equilibrium equations. If there are dependent constraints, the overdeterminacy has to be resolved first by declaring some prescribed generalized strains 
as dependent and putting the dual generalized stress equal to zero. The statically overdetermined force distribution can then be determined at a later stage. The equilibrium equations are obtained from the principle of virtual work.

The equation for the reaction forces $f^{\mathrm{p}}(4)$ is explicit, so if all other variables are known, the reaction forces can directly be obtained from this equation. It should be noted that this is the total force, so if also an applied force is present, the true reaction force is obtained by subtracting this applied force. The other four sets of equations provide the set of non-linear equations for the numerical solution scheme, which has exactly the same number of equations as there are unknowns, which are $\boldsymbol{x}^{\mathrm{f}}, \boldsymbol{\varepsilon}^{\mathrm{f}}, \boldsymbol{\sigma}^{\mathrm{p}}$ and $\boldsymbol{\sigma}^{\mathrm{f}}$. These are solved by a Newton-Raphson iteration. The iteration matrix $\boldsymbol{A}$ is found by taking partial derivatives of the residuals with respect to the unknowns. This yields the set of equations

$$
\boldsymbol{A}\left(\boldsymbol{p}^{(k+1)}-\boldsymbol{p}^{(k)}\right)=-\boldsymbol{r}
$$

where

$$
\boldsymbol{A}=\left[\begin{array}{cccc}
\boldsymbol{K}^{\mathrm{G}} & \boldsymbol{O} & \left(\mathscr{D}_{x, x}^{\mathrm{pf}}\right)^{\mathrm{T}} & \left(\mathscr{D}_{, x}^{\mathrm{ff}}\right)^{\mathrm{T}} \\
\boldsymbol{O} & \boldsymbol{S}^{\mathrm{ff}} & \boldsymbol{O} & -\boldsymbol{I} \\
\mathscr{D}_{, x}^{\mathrm{pf}} & \boldsymbol{O} & \boldsymbol{O} & \boldsymbol{O} \\
\mathscr{D}_{, x}^{\mathrm{ff}} & -\boldsymbol{I} & \boldsymbol{O} & \boldsymbol{O}
\end{array}\right], \quad \boldsymbol{p}=\left[\begin{array}{c}
\boldsymbol{x}^{\mathrm{f}} \\
\boldsymbol{\varepsilon}^{\mathrm{f}} \\
\boldsymbol{\sigma}^{\mathrm{p}} \\
\boldsymbol{\sigma}^{\mathrm{f}}
\end{array}\right]
$$

and the residuals are

$$
\boldsymbol{r}=\left[\begin{array}{c}
\left(\mathscr{D}_{, \boldsymbol{x}}^{\mathrm{pf}}\right)^{\mathrm{T}} \boldsymbol{\sigma}^{\mathrm{p}}+\left(\mathscr{D}_{, \boldsymbol{x}}^{\mathrm{ff}}\right)^{\mathrm{T}} \boldsymbol{\sigma}^{\mathrm{f}}-\boldsymbol{f}^{\mathrm{f}} \\
\boldsymbol{S}^{\mathrm{fp}} \boldsymbol{\varepsilon}^{\mathrm{p}}+\boldsymbol{S}^{\mathrm{ff}} \boldsymbol{\varepsilon}^{\mathrm{f}}+\boldsymbol{\sigma}_{0}^{\mathrm{f}}-\boldsymbol{\sigma}^{\mathrm{f}} \\
\mathscr{D}^{\mathrm{p}}\left(\boldsymbol{x}^{\mathrm{f}}, \boldsymbol{x}^{\mathrm{p}}\right)-\boldsymbol{\varepsilon}^{\mathrm{p}} \\
\mathscr{D}^{\mathrm{f}}\left(\boldsymbol{x}^{\mathrm{f}}, \boldsymbol{x}^{\mathrm{p}}\right)-\boldsymbol{\varepsilon}^{\mathrm{f}}
\end{array}\right] .
$$

Here, the geometric stiffness matrix $\boldsymbol{K}^{\mathrm{G}}$ appears. Formally, it is determined as

$$
\boldsymbol{K}^{\mathrm{G}}=\frac{\partial}{\partial \boldsymbol{x}^{\mathrm{f}}}\left[\left(\mathscr{D}_{, \boldsymbol{x}}^{\mathrm{pf}}\right)^{\mathrm{T}} \boldsymbol{\sigma}^{\mathrm{p}}+\left(\mathscr{D}_{, \boldsymbol{x}}^{\mathrm{ff}}\right)^{\mathrm{T}} \boldsymbol{\sigma}^{\mathrm{f}}-\boldsymbol{f}^{\mathrm{f}}\right]
$$

where the stresses are independent variables and the derivatives of the applied forces yield some terms different from zero because of gravity forces, which may depend on the orientation of elements, applied moments and contributions from the fluid flow of tube elements, see [11]. The equations are solved by a direct solver using Gaussian elimination with partial pivoting for general matrices, which makes a limited use of the sparsity of the iteration matrix. The unknowns in the next iteration numbered $k+1$ are obtained from the ones of the previous iteration numbered $k$ as

$$
\boldsymbol{p}^{(k+1)}=\boldsymbol{p}^{(k)}-\boldsymbol{A}^{-1} \boldsymbol{r}
$$

The iteration is stopped if the corrections are sufficiently small.

\section{Equations of Motion Linearized About a Static Configuration}

The linearized equations for the equilibrium positions can be derived more easily than for the general case. As a first step, the first-order transfer functions have to be determined. The transfer functions give the nodal coordinates and the generalized strains depending on the degrees of freedom. The degrees of freedom are partitioned into prescribed degrees of freedom, $\boldsymbol{q}^{\mathrm{m}}$, which are explicit functions of time, and dynamic degrees of freedom, $\boldsymbol{q}^{\mathrm{d}}$. For static solutions, the prescribed degrees of freedom have constant values, but can themselves be parameters that vary during a continuation, as described in the next section. The transfer functions, also called zero-order transfer functions, for the nodal coordinates and the generalized strains are respectively

$$
\boldsymbol{x}^{\mathrm{f}}=\mathscr{F}\left(\boldsymbol{q}^{\mathrm{m}}, \boldsymbol{q}^{\mathrm{d}}\right), \quad \boldsymbol{\varepsilon}^{\mathrm{f}}=\mathscr{E}\left(\boldsymbol{q}^{\mathrm{m}}, \boldsymbol{q}^{\mathrm{d}}\right)
$$

The transfer functions for the prescribed coordinates and generalized strains are trivial and are not included here. The dependent variables are obtained as a result of the iterative process to determine the equilibrium solution. Virtual variations of the nodal coordinates and the generalized strains are then given by

$$
\delta \boldsymbol{x}^{\mathrm{f}}=\mathscr{F}_{, \boldsymbol{q}^{\mathrm{d}}} \delta \boldsymbol{q}^{\mathrm{d}}, \quad \delta \boldsymbol{\varepsilon}^{\mathrm{f}}=\mathscr{E}_{, \boldsymbol{q}^{\mathrm{d}}} \delta \boldsymbol{q}^{\mathrm{d}}
$$

and actual variations by

$$
\Delta x^{\mathrm{f}}=\mathscr{F}_{, q^{\mathrm{d}}} \Delta \boldsymbol{q}^{\mathrm{d}}, \quad \Delta \boldsymbol{\varepsilon}^{\mathrm{f}}=\mathscr{E}_{, \boldsymbol{q}^{\mathrm{d}}} \Delta \boldsymbol{q}^{\mathrm{d}} .
$$


Their time derivatives at static solutions are given by

$$
\Delta \dot{\boldsymbol{x}}^{\mathrm{f}}=\mathscr{F}_{, \boldsymbol{q}^{\mathrm{d}}} \Delta \dot{\boldsymbol{q}}^{\mathrm{d}}, \quad \Delta \dot{\boldsymbol{\varepsilon}}^{\mathrm{f}}=\mathscr{E}_{, \boldsymbol{q}^{\mathrm{d}}} \Delta \dot{\boldsymbol{q}}^{\mathrm{d}}, \quad \Delta \ddot{\boldsymbol{x}}^{\mathrm{f}}=\mathscr{F}_{, \boldsymbol{q}^{\mathrm{d}}} \Delta \ddot{\boldsymbol{q}}^{\mathrm{d}}, \quad \Delta \ddot{\boldsymbol{\varepsilon}}^{\mathrm{f}}=\mathscr{E}_{, \boldsymbol{q}^{\mathrm{d}}} \Delta \ddot{\boldsymbol{q}}^{\mathrm{d}} .
$$

The mass matrix is never difficult to calculate and is already used for the direct dynamic simulation. After a system mass matrix $\boldsymbol{M}^{\mathrm{ff}}$ has been determined from the contributions of the elements and the nodes, the reduced mass matrix for the degrees of freedom is obtained as

$$
\boldsymbol{M}^{\mathrm{dd}}=\mathscr{F}_{, \boldsymbol{q}^{\mathrm{d}}}^{\mathrm{T}} \boldsymbol{M}^{\mathrm{ff}} \mathscr{F}_{, \boldsymbol{q}^{\mathrm{d}}} .
$$

The material damping terms are collected in system matrix $\boldsymbol{D}^{\mathrm{ff}}$ for the strains and the damping terms from the tube elements acting on the nodal coordinates are collected in another damping matrix $\boldsymbol{C}^{\mathrm{ff}}$. The material damping matrix in the dynamic degrees of freedom is obtained as

$$
D^{\mathrm{dd}}=\mathscr{E}_{, q^{\mathrm{d}}}^{\mathrm{T}} \boldsymbol{D}^{\mathrm{ff}} \mathscr{E}_{, q^{\mathrm{d}}}
$$

and the part resulting from the nodal coordinates is

$$
C^{\mathrm{dd}}=\mathscr{F}_{, q^{\mathrm{d}}}^{\mathrm{T}} \boldsymbol{C}^{\mathrm{ff}} \mathscr{F}_{, q^{\mathrm{d}}}
$$

The stiffness matrix can be obtained from the system geometric stiffness matrix $\boldsymbol{K}^{\mathrm{G}}$ and the system stiffness matrix resulting from the constitutive relations $S$. These result in a reduced material stiffness matrix

$$
\boldsymbol{K}^{\mathrm{dd}}=\mathscr{E}_{, q^{\mathrm{d}}}^{\mathrm{T}} \boldsymbol{S} \mathscr{E}_{, q^{\mathrm{d}}}
$$

and a reduced geometric stiffness matrix

$$
\boldsymbol{G}^{\mathrm{dd}}=\mathscr{F}_{, \boldsymbol{q}^{\mathrm{d}}}^{\mathrm{T}} \boldsymbol{K}^{\mathrm{G}} \mathscr{F}, \boldsymbol{q}^{\mathrm{d}} \cdot
$$

This geometric stiffness matrix differs from the geometric stiffness matrix as customary defined, because the matrix $\boldsymbol{K}^{\mathrm{G}}$ contains also terms from the derivatives of the forces, as seen in Eq. (12), which are normally included in the stiffness matrix resulting from general non-linear terms, $\boldsymbol{N}^{\mathrm{dd}}$, which is not present in the current formulation. As these terms stem from the derivatives of forces with respect to coordinates and not from physical non-linearities, it is natural to include them in the geometric stiffness matrix.

With the reduced matrices, the linearized equations in the dynamic degrees of freedom become

$$
\boldsymbol{M}^{\mathrm{dd}} \Delta \ddot{\boldsymbol{q}}^{\mathrm{d}}+\left(\boldsymbol{C}^{\mathrm{dd}}+\boldsymbol{D}^{\mathrm{dd}}\right) \Delta \dot{\boldsymbol{q}}^{\mathrm{d}}+\left(\boldsymbol{K}^{\mathrm{dd}}+\boldsymbol{G}^{\mathrm{dd}}\right) \Delta \boldsymbol{q}^{\mathrm{d}}=\mathbf{0} .
$$

These linearized equations can be used to calculate the eigenfrequencies and damping ratios with the corresponding modes, which determine the linear stability properties.

In a method in which the equilibrium equations are formulated in the degrees of freedom, these equations are given by

$$
\mathscr{E}_{, \boldsymbol{q}^{\mathrm{d}}}^{\mathrm{T}} \boldsymbol{\sigma}^{\mathrm{f}}-\mathscr{F}_{, \boldsymbol{q}^{\mathrm{d}}}^{\mathrm{T}} \boldsymbol{f}^{\mathrm{f}}=\mathbf{0}
$$

where $\boldsymbol{\sigma}^{\mathrm{f}}$ is given by Eq. (6). The iteration equations in the Newton-Raphson scheme are now given by

$$
\left(\boldsymbol{K}^{\mathrm{dd}}+\boldsymbol{G}^{\mathrm{dd}}\right)\left(\boldsymbol{q}^{\mathrm{d}(k+1)}-\boldsymbol{q}^{\mathrm{d}(k)}\right)=-\mathscr{E}_{, \boldsymbol{q}^{\mathrm{d}}}^{\mathrm{T}} \boldsymbol{\sigma}^{\mathrm{f}}+\mathscr{F}_{, \boldsymbol{q}^{\mathrm{d}}}^{\mathrm{T}} \boldsymbol{f}^{\mathrm{f}},
$$

from which the new approximation $\boldsymbol{q}^{\mathrm{d}(k+1)}$ can be obtained. Note that the dependent variables have to be determined in an iterative way for this method, so in every iteration step for the calculation of the equilibrium solution, an internal iteration has to be performed for the dependent variables.

\section{Continuation of Static Solutions}

It is often desired to know how a static solution changes if a parameter of the system is changed. This can be the case for showing a branch of solutions for a varying design parameter, but here, mainly loading parameters are considered. The solution with the linearized equations can then be represented as a curve depending on the parameter, but often, the solution for the final value of the loading cannot be determined directly in one step because of strong non-linearities in the system response and a step-by-step application of the loading is the way to proceed. Another difficulty is the occurrence of limit points, or turning points, where the loading reaches a maximum and then decreases further on along the solution curve.

Here, six kinds of loading parameters are considered: applied forces or moments, gravity, fluid flow in tube elements, initial stresses, input motion for nodal coordinates and input motion for generalized strains. An initial loading is assumed and then an additional loading can be applied. The parameter, denoted by $\mu$, runs from zero for the initial loading to one for the final, desired loading with the full additional loading. 


\subsection{Description of the Arc Continuation Method}

In an arc continuation method, it is assumed that for a given set of parameter values, a solution has been found in some way. Some previously obtained solutions for different parameter values may or may not be available. A new solution some distance away from the current solution in the space formed by the Cartesian product of the one-dimensional parameter space and the solution space is sought. A metric in this Cartesian product space is defined and a step size $\Delta s$ which approximately measures the distance between the present solution and the new solution is selected. Here, a weighted Cartesian distance is used as a metric, that is,

$$
(\Delta s)^{2}=(\Delta \mu)^{2}+W_{x}^{2}\left[\left\|\Delta \boldsymbol{x}^{\mathrm{f}}\right\|^{2}+\left\|\Delta \boldsymbol{\varepsilon}^{\mathrm{f}}\right\|^{2}\right]+W_{\sigma}^{2}\left[\left\|\Delta \boldsymbol{\sigma}^{\mathrm{f}}\right\|^{2}+\left\|\Delta \boldsymbol{\sigma}^{\mathrm{p}}\right\|^{2}\right],
$$

where $W_{x}$ and $W_{\sigma}$ are positive weight factors. The typical scales of the displacements, strains and stresses with respect to the scale of the parameter are then the reciprocals of the weight factors. Of course, more involved metrics in which each variable has its own weight could be used.

A step in an arc continuation method consists of two parts: the first is a predictor that is used as an initial approximation of the next solution and the second is the corrector part in which a solution is iteratively determined. For this corrector part, a version proposed by Crisfield [8] is chosen, which imposes a constraint on the actual step size. The equations are written for the case in which all weight factors are equal to one; the equations for the case of weight factors not equal to one then easily follow by multiplying and dividing rows and columns of matrices by these factors. Besides the equations (5), (6), (7) and (8), also the equation

$$
(\Delta \mu)^{2}+\|\Delta \boldsymbol{p}\|^{2}-(\Delta s)^{2}=0
$$

has to be satisfied, where it must be understood that the equations may depend on the parameter $\mu$. Instead of Eq. (9), the set of equations that has to be used in the iteration becomes

$$
\left[\begin{array}{cc}
\boldsymbol{A} & \partial \boldsymbol{r} / \partial \mu \\
2 \Delta \boldsymbol{p}^{\mathrm{T}} & 2 \mu
\end{array}\right]\left[\begin{array}{c}
\boldsymbol{p}^{(k+1)}-\boldsymbol{p}^{(k)} \\
\boldsymbol{\mu}^{(k+1)}-\boldsymbol{\mu}^{(k)}
\end{array}\right]=-\left[\begin{array}{c}
\boldsymbol{r} \\
(\Delta \mu)^{2}+\|\Delta \boldsymbol{p}\|^{2}-(\Delta s)^{2}
\end{array}\right] .
$$

If the iteration fails to converge, the chosen step size $\Delta s$ is apparently too large and the step is retried with a halved step size. If the step size is already at its minimum allowed value, the arc continuation has failed at this step and is stopped. If the iteration has converged, a tangent vector to the arc can be determined by finding a solution for the tangent vector $\left[\boldsymbol{t}_{p}^{\mathrm{T}}, t_{\mu}\right]^{\mathrm{T}}$ to the equations

For instance, if $\boldsymbol{A}$ is not singular,

$$
\boldsymbol{A t} \boldsymbol{t}_{p}+(\partial \boldsymbol{r} / \partial \mu) t_{\mu}=\mathbf{0}
$$

$$
t_{\mu}=1, \quad \boldsymbol{t}_{p}=-\boldsymbol{A}^{-1}(\partial \boldsymbol{r} / \partial \mu)
$$

Then the tangent vector is normalized to yield a unit length and the sign is possibly adjusted if the tangent vector does not point in a positive direction along the arc of solutions. This means that for the initial point, $t_{\mu}>0$ and for subsequent points, the scalar product of the tangent vector and the tangent vector of the previous step has to be positive. More generally, a condition is imposed on the cosine, that is, the scalar product, of two consecutive tangent vectors, to be greater than some positive minimum value. If this condition is violated, the step is rejected and a smaller step size is chosen.

Now we can describe the predictor step. For the first step, a linear extrapolation from the initial point is chosen,

$$
\boldsymbol{p}_{1}^{(0)}=\boldsymbol{p}_{0}+\left(\boldsymbol{t}_{p}\right)_{0}(\Delta s)_{1}, \quad \mu_{1}^{(0)}=\mu_{0}+\left(t_{\mu}\right)_{0}(\Delta s)_{1},
$$

where the subscripts 0 and 1 denote variables at the initial point and the first point, respectively. For subsequent points, a cubic extrapolation is used, which makes use of the two previously obtained solutions and the tangent vectors at these points. This is to say,

$$
\begin{aligned}
\boldsymbol{p}_{n}^{(0)} & =\boldsymbol{p}_{n-2}\left(3 \xi^{2}+2 \xi^{3}\right)+\left(\boldsymbol{t}_{p}\right)_{n-2}\left(\xi^{2}+\xi^{3}\right)(\Delta s)_{n-1} \\
& +\boldsymbol{p}_{n-1}\left(1-3 \xi^{2}-2 \xi^{3}\right)+\left(\boldsymbol{t}_{p}\right)_{n-1}\left(\xi+2 \xi^{2}+\xi^{3}\right)(\Delta s)_{n-1}, \\
\mu_{n}^{(0)} & =\mu_{n-2}\left(3 \xi^{2}+2 \xi^{3}\right)+\left(t_{\mu}\right)_{n-2}\left(\xi^{2}+\xi^{3}\right)(\Delta s)_{n-1} \\
& +\mu_{n-1}\left(1-3 \xi^{2}-2 \xi^{3}\right)+\left(t_{\mu}\right)_{n-1}\left(\xi+2 \xi^{2}+\xi^{3}\right)(\Delta s)_{n-1},
\end{aligned}
$$

where the subscripts $n, n-1$ and $n-2$ denote variables at the predictor point, the current point and the previous point, respectively, and $\xi=(\Delta s)_{n} /(\Delta s)_{n-1}$ is the ratio of the current step size and the previous step size. This cubic extrapolation can already be found in [4], where it is attributed to Shearing. 
After a successful step, a new step size for the next step has to be chosen. It is chosen as

$$
(\Delta s)_{n+1}=(\Delta s)_{n} \min \left(\frac{1}{2} \sqrt{\frac{1-\cos \beta_{\mathrm{max}}}{1-\cos \beta_{n}}}, \frac{n_{\mathrm{it}, \mathrm{opt}}^{2}}{n_{\mathrm{it}, n}^{2}}\right) .
$$

Here, $\beta_{\max }$ is the maximum allowed angle between two consecutive tangent vectors, $\beta_{n}$ is the actual angle between the tangent vectors at steps $n-1$ and $n, n_{\mathrm{it}, \mathrm{opt}}$ is the assumed optimal number of iterations in the corrector step and $n_{\mathrm{it}, n}$ is the actual number of iterations in step $n$. A further restriction is that the step size must be between the minimum and the maximum allowed value, which is enforced if the step size as determined above happens to be outside this interval, by pushing it back to the nearest allowed value.

\subsection{Calculation of Derivatives with Respect to the Parameter}

In the above equations, it was not specified how the derivatives $\partial \boldsymbol{r} / \partial \mu$ can be calculated for the different kinds of loadings. Here, we limit ourselves for the time being to the six kinds of loadings mentioned earlier.

For the case that the loading is the magnitude of an applied force or an applied moment in the planar case, the derivatives are

$$
\frac{\partial \boldsymbol{r}}{\partial \mu}=\left[\begin{array}{c}
-\left(\boldsymbol{f}_{\text {end }}^{\mathrm{f}}-\boldsymbol{f}_{\text {begin }}^{\mathrm{f}}\right) \\
\mathbf{0} \\
\mathbf{0} \\
\mathbf{0}
\end{array}\right]
$$

and for the case that the loading is the magnitude of an applied moment with a fixed direction in space for the spatial case, we have for the derivative of the force vector restricted to the node on which the moment $\boldsymbol{T}$ is applied

$$
-\frac{\partial \boldsymbol{f}^{(\lambda)}}{\partial \mu}=-2 \boldsymbol{\Lambda}^{\mathrm{T}}\left(\boldsymbol{T}_{\mathrm{end}}-\boldsymbol{T}_{\text {begin }}\right)
$$

where $\boldsymbol{f}^{(\lambda)}$ are the force component dual to the Euler parameters $\lambda=\left[\lambda_{0}, \lambda_{1}, \lambda_{2}, \lambda_{3}\right]^{\mathrm{T}}$ and the matrix $\boldsymbol{\Lambda}$ is defined as

$$
\Lambda=\left[\begin{array}{rrrr}
-\lambda_{1} & \lambda_{0} & -\lambda_{3} & \lambda_{2} \\
-\lambda_{2} & \lambda_{3} & \lambda_{0} & -\lambda_{1} \\
-\lambda_{3} & -\lambda_{2} & \lambda_{1} & \lambda_{0}
\end{array}\right]
$$

For the case that the loading is the acceleration of gravity, we first consider the way in which the gravity forces are included in the direct analysis. An acceleration of gravity $g$ can be seen as an acceleration of the reference frame with the same magnitude, but in the opposite direction. This means that all coordinates representing positions can be thought to have an acceleration corresponding to this opposite direction, and all coordinates representing rotations are unaffected. If we collect all the accelerations of gravity in a vector denoted by $\boldsymbol{a}_{g}$, the forces of gravity are given by

$$
M a_{g}
$$

The corresponding derivative is given by

$$
\frac{\partial \boldsymbol{r}}{\partial \mu}=\left[\begin{array}{c}
-\boldsymbol{M}^{\mathrm{fp}}\left(\boldsymbol{a}_{g, \text { end }}^{\mathrm{p}}-\boldsymbol{a}_{g, \text { begin }}^{\mathrm{p}}\right)-\boldsymbol{M}^{\mathrm{ff}}\left(\boldsymbol{a}_{g, \text { end }}^{\mathrm{f}}-\boldsymbol{a}_{g, \text { begin }}^{\mathrm{f}}\right) \\
\mathbf{0} \\
\mathbf{0} \\
\mathbf{0}
\end{array}\right]
$$

Note that also the accelerations at points that are fixed or have prescribed displacements have to be taken into account.

We next consider the case that the loading is a pre-stress, that is, a prescribed stress associated with a free deformation, in the same way as a force on a nodal point can be prescribed. Note that additional stresses due to stiffness can be present, which are added to the prescribed stresses. If we denote the prescribed stresses by $\boldsymbol{\sigma}_{0}$, the derivatives are

$$
\frac{\partial \boldsymbol{r}}{\partial \mu}=\left[\begin{array}{c}
\mathbf{0} \\
\left(\boldsymbol{\sigma}_{0, \text { end }}^{\mathrm{f}}-\boldsymbol{\sigma}_{0, \text { begin }}^{\mathrm{f}}\right) \\
\mathbf{0} \\
\mathbf{0}
\end{array}\right]
$$


The flows of the tube elements give a contribution to the force, which is, in the static case, proportional to the square of the mass flow. If we denote this force of an element by $\boldsymbol{f}_{\text {flow }}^{e}$ and the mass flow of an element by $\dot{m}^{e}$, the derivative of this force with respect to the parameter is given by

$$
\frac{\partial \boldsymbol{f}_{\mathrm{flow}}^{e}}{\partial \mu}=2 \frac{\boldsymbol{f}_{\mathrm{flow}}^{e}}{\dot{m}^{e}}\left(\dot{m}_{\mathrm{end}}^{e}-\dot{m}_{\mathrm{begin}}^{e}\right) .
$$

The derivatives of the individual tube elements associated with free coordinates can be assembled in the first subvector of the derivative of the residual vector with respect to the parameter $\mu$.

Finally, the derivatives due to variations in the input motion need to be determined. We can define a geometric stiffness matrix $\boldsymbol{K}^{\mathrm{G} . p}$ associated with the prescribed coordinates in the same way as the geometric stiffness matrix $\boldsymbol{K}^{\mathrm{G}}$ associated with the free coordinates, as defined in Eq. (12), as

$$
\boldsymbol{K}^{\mathrm{G}, \mathrm{p}}=\frac{\partial}{\partial \boldsymbol{x}^{\mathrm{p}}}\left[\left(\mathscr{D}_{, \boldsymbol{x}}^{\mathrm{pf}}\right)^{\mathrm{T}} \boldsymbol{\sigma}^{\mathrm{p}}+\left(\mathscr{D}_{, \boldsymbol{x}}^{\mathrm{ff}}\right)^{\mathrm{T}} \boldsymbol{\sigma}^{\mathrm{f}}-\boldsymbol{f}^{\mathrm{f}}\right]
$$

Now the derivative of the residual vector with respect to the parameter is

$$
\frac{\partial \boldsymbol{r}}{\partial \mu}=\left[\begin{array}{c}
\boldsymbol{K}^{\mathrm{G}, \mathrm{p}}\left(\boldsymbol{x}_{\text {end }}^{\mathrm{p}}-\boldsymbol{x}_{\text {begin }}^{\mathrm{p}}\right) \\
\boldsymbol{S}^{\mathrm{fp}}\left(\boldsymbol{\varepsilon}_{\mathrm{end}}^{\mathrm{p}}-\boldsymbol{\varepsilon}_{\text {begin }}^{\mathrm{p}}\right) \\
\mathscr{D}_{, \boldsymbol{x}}^{\mathrm{pp}}\left(\boldsymbol{x}_{\text {end }}^{\mathrm{p}}-\boldsymbol{x}_{\text {begin }}^{\mathrm{p}}\right)-\left(\boldsymbol{\varepsilon}_{\text {end }}^{\mathrm{p}}-\boldsymbol{\varepsilon}_{\text {begin }}^{\mathrm{p}}\right) \\
\mathscr{D}_{, \boldsymbol{x}}^{\mathrm{fp}}\left(\boldsymbol{x}_{\text {end }}^{\mathrm{p}}-\boldsymbol{x}_{\text {begin }}^{\mathrm{p}}\right)
\end{array}\right] .
$$

If there are several loadings of different kinds that need to be varied, the contributions of the individual loadings can be added.

\section{Applications and Comparisons}

\subsection{Large Deflections of Beams}

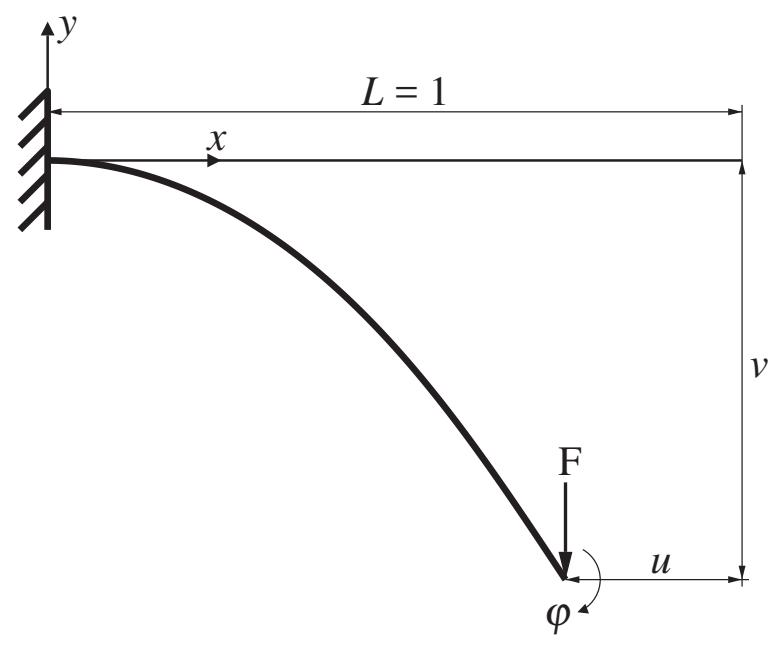

Figure 1: Planar cantilever beam subjected to a transverse tip force. Shown is the deflection for a scaled force $F=3$.

As a first example, the large deflection of a planar cantilever beam loaded by a transverse tip force is studied, see Figure 1. The length of the beam is scaled to unity, as is its flexural rigidity and its mass. As the cross-section is square with a height-to-length ratio of 0.05 , the scaled normal stiffness is 4800 . The beam is modelled with eight equal planar beam elements with a length of 0.125 . The degrees of freedom can be chosen as the generalized strains of the beams, three for each one, or as the nodal coordinates of the free nodes, also three for each node. This gives 24 degrees of freedom of the complete system in both cases. First, the maximum loading that can be applied in a single step still giving a converged solution is determined. The results are given in Table 1. It is seen that the choice of the degrees of freedom has no influence on the convergence, as could be expected, because the same system of equations has to be 


\begin{tabular}{|l|c|c|}
\hline Choice of the degrees of freedom & Method & Maximum loading step \\
\hline Generalized strains & Minimal set of equations & 3.24 \\
Generalized strains & Extended set of equations & 4.49 \\
Nodal coordinates & Minimal set of equations & 3.76 \\
Nodal coordinates & Extended set of equations & 4.49 \\
\hline
\end{tabular}

Table 1: Maximum loading steps for different choices of degrees of freedom and solution methods.

solved in this method. Furthermore, a larger step could be taken than in the cases in which a minimal set of equations is used. The difference in computation time for this small problem is insignificant.

Also for a large tip force of 100 , the deflection is calculated, making use of the arc continuation method. With an initial step size of 4 , optimal number of iterations of $4, \cos \beta_{\max }=0.9$, and a weight factor of 0.1 for the generalized forces and stresses, 13 steps were needed to traverse the path. The deflections and rotations at the tip for the final loading are $u=0.8833, v=0.9754$ and $\varphi=1.5707$. It should be noted that for eight elements and this large tip force, the final results are not accurate, but this example shows the possibilities of the arc continuation method.

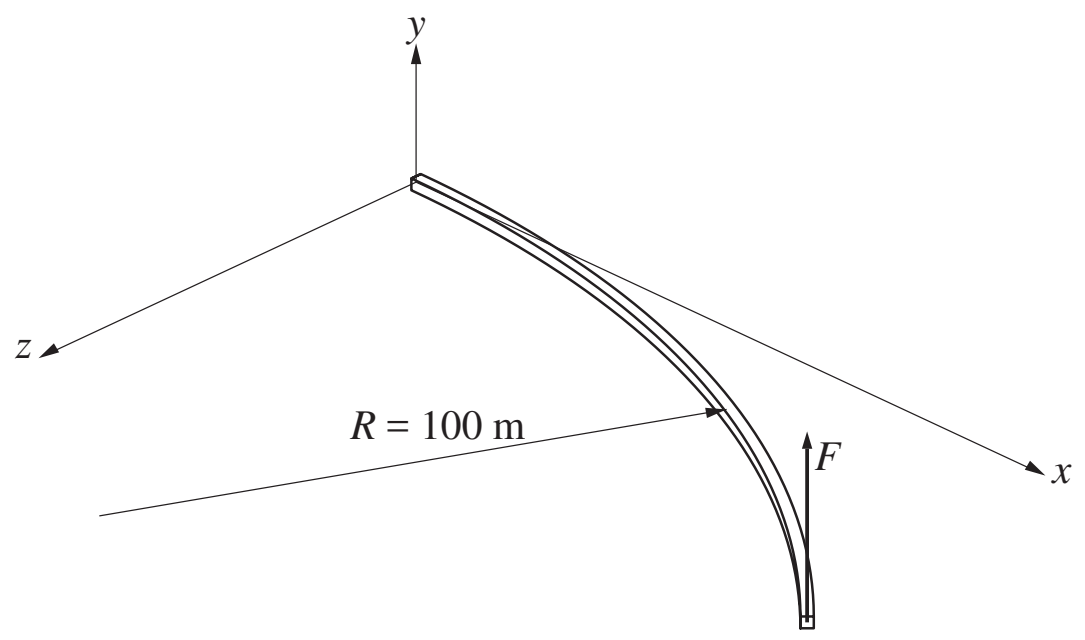

Figure 2: Curved spatial cantilever beam subjected to a tip force perpendicular to plane of the curve.

A second example concerns a spatial cantilever beam bent over 45 degrees and loaded by a force at its tip in a direction perpendicular to the plane of the bend, see Figure 2. The radius of the curve is $100 \mathrm{~m}$, so the total length of the beam is $25 \pi \mathrm{m}$. The cross-section has dimensions of $1 \mathrm{~m}$ by $1 \mathrm{~m}$, the normal rigidity is $1 \times 10^{7} \mathrm{~N}$ and the flexural rigidity in both directions, as well as a torsional stiffness, is $1 \times 10^{7} / 12 \mathrm{Nm}^{2}$. Effects of shear deformation and the warping of cross-sections are left out of consideration. The force of $600 \mathrm{~N}$ is applied in four steps of $150 \mathrm{~N}$ each. The bend is approached by a number of straight beam elements which are connected at an angle in such a way that the nodal points are on the centre line of the curved beam. The generalized strains of the elements are chosen as the degrees of freedom, six for each element. The positions of the tip for the largest force for models with different numbers of elements are shown in Table 2. The results for eight elements agree with those given in [12]. For both solution methods, about the same number of iterations are needed, but the proposed method with an extended system of equations performs better, and more so if the number of elements is increased. For the case of 16 elements, the proposed method is about four times as fast.

\begin{tabular}{|c|c|c|c|}
\hline Number of elements & $x_{\text {tip }}$ in $\mathrm{m}$ & $y_{\text {tip }}$ in $\mathrm{m}$ & $z_{\text {tip }}$ in $\mathrm{m}$ \\
\hline 2 & 43.130 & 56.547 & 14.694 \\
4 & 46.286 & 54.166 & 15.487 \\
8 & 46.944 & 53.639 & 15.639 \\
16 & 47.086 & 53.497 & 15.757 \\
\hline
\end{tabular}

Table 2: Tip position $\left(x_{\text {tip }}, y_{\text {tip }}, z_{\text {tip }}\right)$ for a tip force $F=600 \mathrm{~N}$ and models with different numbers of elements. 


\subsection{Buckling of a Parallel Leaf-Spring Guidance Due to Misalignment}

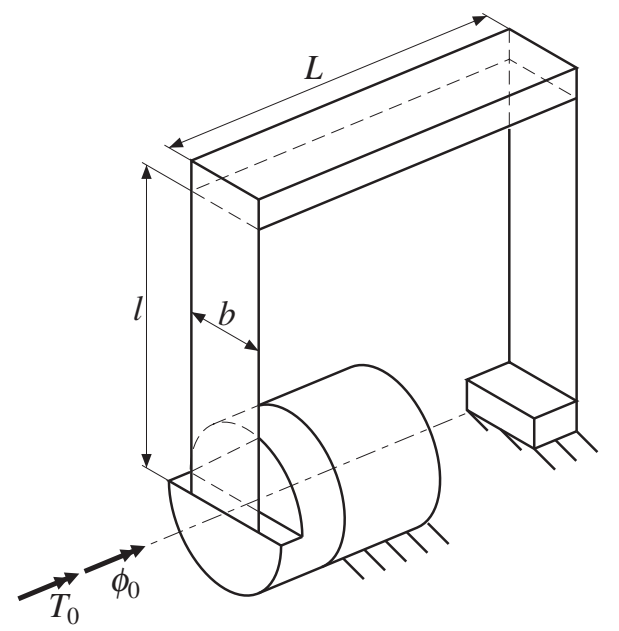

Figure 3: Parallel leaf-spring guidance with an adjustable misalignment.

A parallel leaf-spring guidance as shown in Figure 3 consists of a fixed base, a shuttle and two leaf-springs which connect the shuttle to the base. The shuttle has a low stiffness in one direction, a translation perpendicular to the planes of the leaf-springs, and a high stiffness in all complementary directions. By a hinge for which the rotation or the applied moment can be prescribed, small misalignments in the stiff direction or a pre-stress can be introduced in the mechanism. If the misalignment or the pre-stress is high enough, the leaf-springs may buckle. The length $l$, the width $b$, the thickness $t$, Young's modulus $E$, Poisson's ratio $v$ and the mass density $\rho$ of the leaf-springs as well as the distance $L$, the acceleration of gravity $g$ and the mass of the shuttle $m_{\mathrm{s}}$ are shown in Table 3 . This model is a simplified version of the model presented earlier [13]. Each leaf-spring is modelled by ten equal beam elements, where the torsional rigidities of the beam elements near the clampings are increased to include the effect of the constrained warping: the effective length is reduced by $b \sqrt{(1+v) / 24}$. No shear in the leaf-springs is taken into account.

\begin{tabular}{|c|l|c|c|}
\hline Symbol & Description & Value & Dimension \\
\hline$l$ & Length of the leaf-springs & 100 & $\mathrm{~mm}$ \\
$b$ & Width of the leaf-springs & 30 & $\mathrm{~mm}$ \\
$t$ & Thickness of the leaf-springs & 0.2 & $\mathrm{~mm}$ \\
$E$ & Young's modulus & 195 & $\mathrm{GPa}$ \\
$v$ & Poison's ratio & 0.29 & - \\
$\rho$ & Mass density & 8000 & $\mathrm{~kg} / \mathrm{m}^{3}$ \\
$L$ & Length of the shuttle & 120 & $\mathrm{~mm}$ \\
$g$ & Acceleration of gravity & 9.81 & $\mathrm{~m} / \mathrm{s}^{2}$ \\
$m_{\mathrm{S}}$ & Mass of the shuttle & 0.1512 & $\mathrm{~kg}$ \\
\hline
\end{tabular}

Table 3: Data for the parallel leaf-spring guidance.

In order to introduce an imperfection, so the solution does not follow the fundamental solution if it becomes unstable, but follows the buckled state of the mechanism, a lateral component of the force of gravity in the direction of the low stiffness of the shuttle is introduced; its magnitude is $\mathrm{g} / 10000$. The chosen degrees of freedom are the generalized strains of the beam elements, whereas six of them are dependent, so the system has $6 \times 20-6=114$ degrees of freedom. The hinge is given a prescribed rotation of $2 \mathrm{mrad}$, which is applied in steps. At an angle of about $0.70 \mathrm{mrad}$, the fundamental solution becomes unstable, and a path of stable buckled states is followed. If the increments in the applied rotation are taken too large, another branch of buckled solutions is found, which are, however, unstable. For the original model, where the equations are expressed in the degrees of freedom, about 3600 equal increments are needed. For the extended system, one could obtain the solution with 800 equal increments, resulting in a reduction of the computational time by about a factor of 30. With the arc continuation method, the correct branch of solutions could be obtained with 235 steps, where the maximum step size had to be chosen sufficiently small to guarantee that the right branch was chosen. With a larger step size, another solution branch with unstable solutions is found. The applied 
moments for both branches are shown in Figure 4. The gain in efficiency with respect to the original formulation is about a factor of 70 .

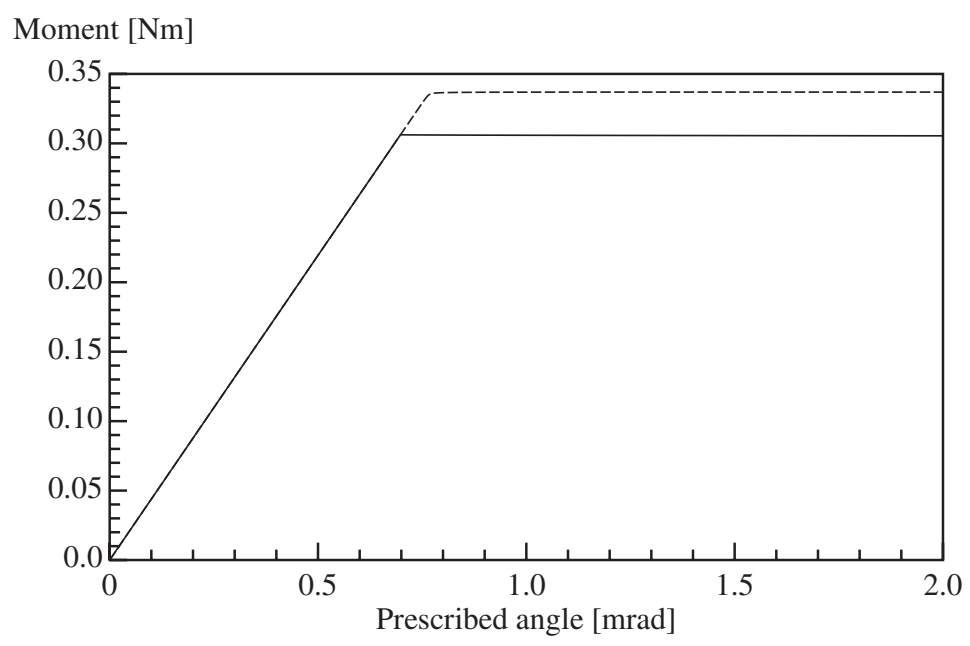

Figure 4: Applied moment $T_{0}$ as a function of the absolute value of the prescribed rotation angle $\phi_{0}$ of the hinge. The fully drawn line represents a stable branch, whereas the dashed line represents an unstable branch of solutions.

\subsection{Large Deflections of a Fluid-Conveying Tube}

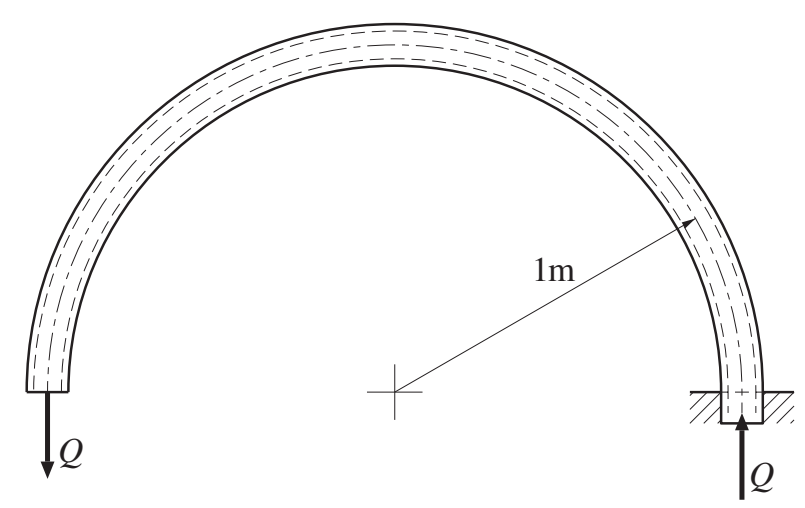

Figure 5: Semicircular tube conveying a fluid.

The last example concerns the static configuration of a flexible fluid-conveying semicircular tube, where the equilibrium position is studied for varying mass flow rates. The system as shown in Figure 5 consists of a tube bent into a half-circle with a radius of $1 \mathrm{~m}$. One end of the tube, where the fluid enters, is held fixed, whereas at the other free end the fluid exits the tube. The mass flow rate is kept constant, but its value can vary. The tube has a mass per unit of length of $0.75 \mathrm{~kg} / \mathrm{m}$ and the fluid has a mass per unit of length of the tube of $0.25 \mathrm{~kg} / \mathrm{m}$, so their combined mass per unit of length is $1 \mathrm{~kg} / \mathrm{m}$. The mass moment of inertia per unit of length of the cross-section about a central transverse axis is $5 \times 10^{-5} \mathrm{kgm}$. The stiffness properties of the tube are a normal stiffness of $1 \times 10^{5} \mathrm{~N}$, a torsional stiffness of $0.8 \mathrm{Nm}^{2}$ and a flexural rigidity of $1 \mathrm{Nm}^{2}$. The mass flow rate varies from 0 to $0.6 \mathrm{~kg} / \mathrm{s}$.

The tube is modelled by twelve equal tube elements. This type of element has been described in [11]. Essentially, the element is a curved beam with a fluid flowing through it. The four bending deformations and the torsional strain are taken as degrees of freedom, whereas the elongation of the beam axis is suppressed; this results in a model with 60 degrees of freedom. The mass flow rate is chosen as the variable parameter.

It appears that for this example, the method which uses a formulation in the degrees of freedom actually performs better in that a larger step size still gives convergence. In fact, an initial increment of $0.825 \mathrm{~kg} / \mathrm{s}$ still results in a 


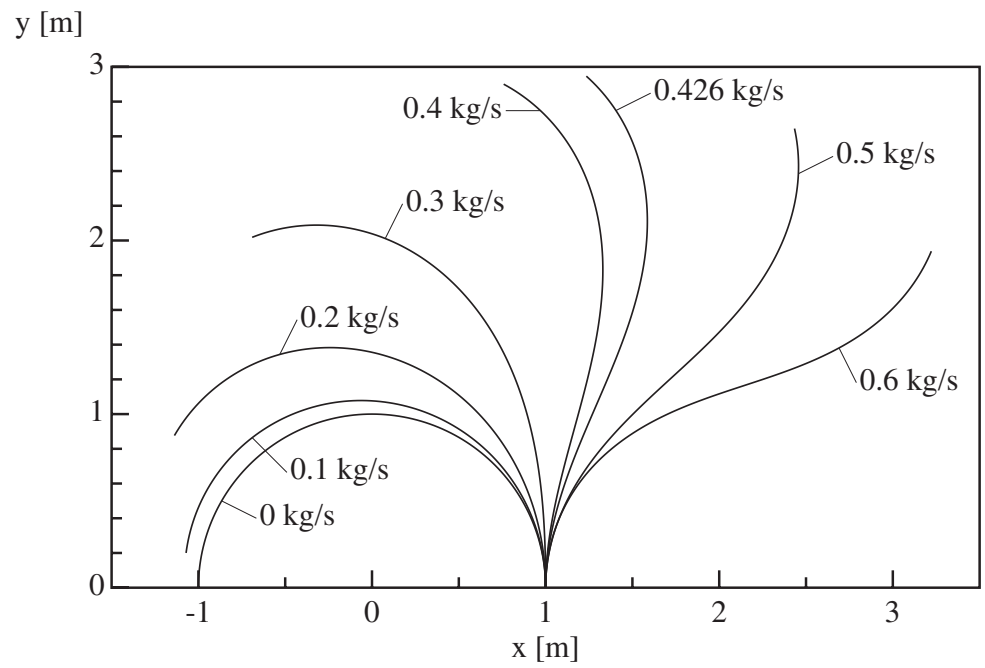

Figure 6: Configirations of the centreline of the tube for flow rates of $0,0.1,0.2,0.3,0.4,0.426,0.5 \mathrm{and} 0.6 \mathrm{~kg} / \mathrm{s}$.

converged solution, whereas for the enlarged system proposed here, no convergence was found for initial steps larger than $0.22 \mathrm{~kg} / \mathrm{s}$. On the other hand, the proposed method requires less computational time per converged step. In fact, quite large deflections of the tube occur: for several mass flow rates, the shape of the centreline of the tube is shown in Figure 6 . For a mass flow rate of about $0.426 \mathrm{~kg} / \mathrm{s}$, the stationary solution of the tube becomes unstable. The instability occurs at a Hopf bifurcation for an out-of-plane mode with a circular frequency of $1.056 \mathrm{rad} / \mathrm{s}$ with a complex mode shape, which is shown in Figure 7. As the mode shape is a complex quantity, the displacements are shown as complex numbers. It can be seen that the tip displacement lags in phase with respect to the displacement close to the inlet. This phase difference causes the input of energy into the motion of the tube if the mass flow rate is further increased.

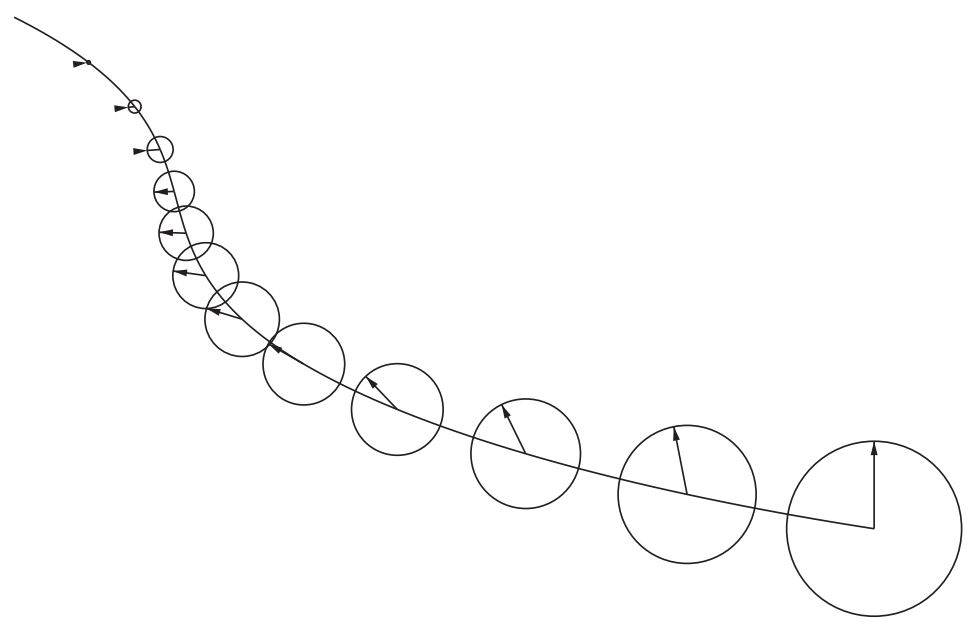

Figure 7: Eigenmode at the critical mass flow of $0.426 \mathrm{~kg} / \mathrm{s}$. The $z$-displacements at the nodes is shown as a complex quantity represented by an arrow in a circle. The deflected shape is shown in a skew-parallel projection.

\section{Conclusions}

The presented method for calculating static solutions for multibody dynamic systems works well for most systems and has an efficiency advantage with respect to a method using a system of equations formulated in a minimal set of coordinates. The method avoids large forces and generalized stresses at initial stages in the iteration process, and therefore the non-linearities to be dealt with are smaller, which explains the increased robustness.

The arc continuation method allows a calculation of a branch of solutions. Especially for branches that pass close by 
bifurcations, the continuation method has its merits. The adaptive step size control and the reduction of the step size if the iteration does not converge to a solution makes the procedure robust, so less user interaction is needed. This makes the procedure especially useful for optimization in which a large number of calculations with different parameter values are needed. It should be remarked that the proposed arc continuation method is rather general and can be applied to other formulations for the equilibrium equations or to reduced-order models.

\section{Acknowledgment}

This research was funded by the Innovative Research Incentives Scheme VIDI (14152 NWO TTW) of the Ministry of Education, Culture and Science of the Netherlands.

\section{References}

[1] K. van der Werff, Kinematic and dynamic analysis of mechanisms, a finite element approach, Dissertation, Delft: Delft University Press, 1977.

[2] K. van der Werff and J. B. Jonker, "Dynamics of flexible mechanisms," in E. J. Haug, Computer Aided Analysis and Optimization of Mechanical System Dynamics, Berlin: Springer-Verlag, pp. 381-400, 1984.

[3] J. B. Jonker, "A finite element dynamic analysis of spatial mechanisms with flexible links," Computer Methods in Applied Mechanics and Engineering, vol. 76, pp. 17-40, 1989.

[4] C. B. Haselgrove, "The solution of non-linear equations and of differential equations with two-point boundary conditions," The Computer Journal, vol. 4, pp. 255-259, 1961.

[5] E. Riks, On the numerical solution of snapping problems in the theory of elastic stability, Dissertation, Stanford: Stanford University, 1970.

[6] E. Riks, "The application of Newton's method to the problem of elastic stability," ASME Journal of Applied Mechanics, vol. 39, pp. 1060-1065, 1972.

[7] G. A. Wempner, "Discrete approximations related to nonlinear theories of solids," International Journal of Solids and Structures, vol. 7, pp. 1581-1599, 1971.

[8] M. A. Crisfield, "A fast incremental/iterative solution procedure that handles "snap-through"," Computers and Structures, vol. 13, pp. 55-62, 1981.

[9] I. Fried, "Orthogonal trajectory accession to the nonlinear equilibrium curve," Computer Methods in Applied Mechanics and Engineering, vol. 47, pp. 283-297, 1984.

[10] A. Cardona and A. Huespe, "Continuation methods for tracing the equilibrium path in flexible mechanism analysis,” Engineering Computations, vol. 15, pp. 190-220, 1998.

[11] J. P. Meijaard, "Fluid-conveying flexible pipes modeled by large-deflection finite elements in multibody systems," ASME Journal of Computational and Nonlinear Dynamics vol. 9, no. 1, 2014.

[12] J. B. Jonker and J. P. Meijaard, "A geometrically non-linear formulation of a three-dimensional beam element for solving large deflection multibody system problems," International Journal of Non-Linear Mechanics, vol. 53, pp. 63-74, 2013.

[13] J. P. Meijaard, D. M. Brouwer and J. B. Jonker, "Analytical and experimental investigation of a parallel leaf spring guidance,” Multibody System Dynamics, vol. 23, pp. 77-97, 2010. 